\title{
Feedback for nonlinear system identification
}

\author{
Thiago Burghi ${ }^{1}$, Maarten Schoukens ${ }^{2}$ and Rodolphe Sepulchre ${ }^{1}$
}

\begin{abstract}
Motivated by neuronal models from neuroscience, we consider the system identification of simple feedback structures whose behaviors include nonlinear phenomena such as excitability, limit-cycles and chaos. We show that output feedback is sufficient to solve the identification problem in a two-step procedure. First, the nonlinear static characteristic of the system is extracted, and second, using a feedback linearizing law, a mildly nonlinear system with an approximately-finite memory is identified. In an ideal setting, the second step boils down to the identification of a LTI system. To illustrate the method in a realistic setting, we present numerical simulations of the identification of two classical systems that fit the assumed model structure.
\end{abstract}

Index Terms-Excitability, Approximately-finite memory, Systems identification, Nonlinear systems, Output feedback

\section{INTRODUCTION}

System identification of nonlinear dynamical systems has been a topic of increasing interest in the recent years, see e.g. [11] [12]. The approach in these references is block-oriented, and finds its roots in specific structures such as WienerHammerstein models [4]. These block-oriented approaches exploit the idea of estimating a best linear approximation [10] of the nonlinear system as a first step in the direction of solving the identification problem. A common underlying assumption in the estimation of approximate linear models is that the system class has some variant of the fading memory property, meaning that the output signals depend on the past of the input signals with a forgetting factor, see e.g. [1] and [8].

The present work seeks to extend the above methods to input-output nonlinear behaviors that can be transformed by output feedback into operators with a fading memory. More specifically, we observe that the simple interconnection structure in Figure 1 possesses that property, by inspection, and is general enough to include nonlinear behaviors that are hard to identify with state-of-the art methods.

In particular, we are motivated by conductance-based models of neurons. Those models, pioneered by Hodgkin and Huxley in their seminal work [2], have become central to neurophysiology and computational neuroscience. Their

*The research leading to these results has received funding from the European Research Council under the Advanced ERC Grant Agreement Switchlet n.670645 and from the Brazilian federal agency for the Coordination of Improvement of Higher Education Personnel (CAPES).

${ }^{1} \mathrm{~T}$. Burghi (tbb29@cam.ac.uk) and R. Sepulchre (r.sepulchredeng.cam.ac.uk) are with the Department of Engineering, Control Group, University of Cambridge, Cambridge CB2 1PZ, UK. ${ }^{2}$ M. Schoukens (m.schoukens@tue.nl) is with the Department of Electrical Engineering, Eindhoven University of Technology, 5612 AZ Eindhoven, Netherlands.

(C)EUCA. Published in: 18th European Control Conference (ECC), Napoli, Italy, June 25-28 2019.

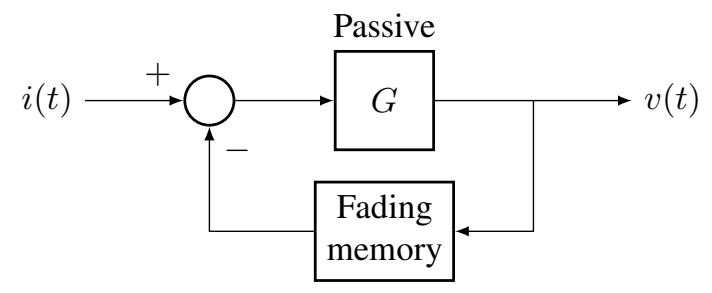

Fig. 1. A nonlinear feedback circuit. In this paper, the fading memory block is a static nonlinearity $h(\cdot)$, and $G$ is LTI.

behaviors include nonlinear phenomena such as excitability, limit cycles, bistability, and bursting. Yet, all conductancebased models share the structure in Figure 1 where the passive element models the passive behavior of the cellular membrane and the fading memory operator models the voltage-gated conductance of ion channels. We advocate that such models can be transformed by feedback into operators with a fading memory, and that this property makes them amenable to rigorous system identification. This property is in fact at the root of the voltage-clamp experiment that has been central to the conductance-based modelling principle over the last seventy years.

As a first step, in this paper, we focus on the elementary situation where the fading memory component in Figure 1 is static, and the passive element is LTI. The feedback structure then becomes the classical structure of a Lure system. This simplified structure already includes famous models such as the excitable circuit of Fitzugh and Nagumo [6] and the chaotic circuit of Chua [5]. We show that the identification of such nonlinear circuits becomes straightforward if we introduce output feedback in experiment design. Not surprisingly, the static element can be identified separately from the LTI element. This allows the use of a feedback linearizing law to transform the identification problem into that of identifying a mildly nonlinear system with an approximately-finite memory [8] - a specific type of fading memory property.

Although elementary, we believe that this methodology is general and appealing for the identification of nonlinear systems that do not have the fading memory property. This methodology is also in line with the idea that smart experiment design is important to obtain good models of nonlinear systems.

The paper is organized in the following way. In Section [II we define the model class which we are interested in identifying, and give two examples of systems that fit in that class. In Section [III, we recall the concept of approximatelyfinite memory, and show that output feedback can be used to endow systems in the defined model class with that 
property. In Section IV we develop the main contribution of the paper, based on a two-step identification procedure for identifying systems in the model class. In Section $\nabla$ we present numerical simulations concerning the identification of the examples from Section $\Pi$ in a realistic setting. Some concluding remarks are presented in Section VI.

\section{MODEL STRUCTURE}

The model and input classes of interest are defined below.

Definition 1 (Model class). We will work with the class of systems given by the negative feedback interconnection between a causal LTI component $G$ and a nonlinear static map $h$, as in Figure 1 The map $h: \mathbb{R} \rightarrow \mathbb{R}$ is a continuous function such that, without loss of generality, $h(0)=0$. In addition, there are two real constants $\rho_{1}$ and $\rho_{2}$ such that

$$
\rho_{1} \leq \frac{h\left(v_{2}\right)-h\left(v_{1}\right)}{v_{2}-v_{1}} \leq \rho_{2}
$$

for all $v_{2} \neq v_{1}$. The LTI component $G$ belongs to the set of real-rational, strictly proper transfer functions $G(s)=$ $N(s) / D(s)$ such that all poles of $G(s)$ are in $\operatorname{Re}[s]<0$, $\operatorname{Re}[G(j \omega)] \geq 0$ for all $\omega \in \mathbb{R}$, and $G(0)>0$.

The above implies that $G(s)$ is positive-real [3, Definition 6.4], and that $\operatorname{deg} D(s)-\operatorname{deg} N(s)=1$. We denote $\|G\|_{1}=$ $\int_{0}^{\infty}|g(t)| d t$, where $g(t)$ is the impulse response of $G(s)$.

Definition 2 (Input class). For an arbitrary $\xi>0$, the input class $\mathcal{U} \subset \mathcal{L}_{\infty}\left(\mathbb{R}_{+}\right)$is the set of functions $u$ from $\mathbb{R}_{+}=$ $[0, \infty)$ to $\mathbb{R}$ such that $\sup _{t \geq 0}|u(t)|<\xi$.

\section{A. Some examples}

In this section, we provide two simple examples of circuits that belong to the model class defined above.

Example 1. The Fitzhugh-Nagumo (FHN) circuit [6] was proposed as a simple model of realistic neurons and became a paradigm of excitability. The model has the state-space representation

$$
\begin{aligned}
\frac{1}{20} \dot{v} & =-x-h(v)+i \\
\dot{x} & =-\frac{3}{4} x+v
\end{aligned}
$$

where $h$ is given by the nonlinear characteristid 1

$$
h(v)=-v+v^{3} / 3
$$

Note that $\rho_{1}=-1$, and the nonlinear resistance is locally active. It can be verified that the system (2)-(3) belongs to the model class of Definition 1, with

$$
G(s)=\frac{20 s+15}{s^{2}+0.75 s+20} .
$$

For $i=0$, the system behaves as an autonomous relaxation oscillator. For $i=-1.5$, the output $v(t)$ converges to a constant equilibrium, and the system is excitable: the output can display high-amplitude excursions away from

\footnotetext{
${ }^{1}$ Note that if $i \in \mathcal{U}$ we can always choose a bounded positively invariant state-space $X$ for this system. Then, $h(v)$ satisfies $(1)$ in $X$. Such a set $X$ can be found, for instance, using the Lyapunov function $V(v, x)=$ $v^{2} / 2+10 x^{2}$ and the standard arguments in [3, Section 4.8].
}

equilibrium, called spikes, when the input $i$ is increased momentarily past a certain excitability threshold [13].

Example 2. The Chua circuit [5] is constructed with two capacitors $c_{1}>0$ and $c_{2}>0$, an inductor $\ell>0$, a resistor $r>0$ and a Chua diode. The Chua diode is a nonlinear resistive element with a piecewise-linear monotonically decreasing characteristic given by

$$
h(v)=\left\{\begin{array}{cc}
-0.1(v+1)+4, & v \leq-1 \\
-4 v, & -1<v<1 \\
-0.1(v-1)-4 & v \geq 1
\end{array}\right.
$$

The passive element of the Chua circuit is given by

$$
G(s)=\frac{\ell c_{2} s^{2}+\ell r s+1}{\ell c_{1} c_{2} s^{3}+\ell r\left(c_{1}+c_{2}\right) s^{2}+c_{1} s+r}
$$

In [5], it is shown that the autonomous Chua circuit presents chaotic behavior when the parameters are given by $c_{1}=0.1, c_{2}=2, \ell=1 / 7$ and $r=0.7$. By forcing the Chua circuit with an external current, the circuit belongs to the model class of Definition 1. Note that in this case $\rho_{1}=-4$ and $\rho_{2}=-0.1$.

\section{APPROXIMATELY-FINITE MEMORY THROUGH OUTPUT FEEDBACK}

In this section, we discuss how the feedback law

$$
i=k\left(v_{\mathrm{r}}-v\right)
$$

is used to endow a system from the model class of Definition 1 with the approximately-finite memory property [9].

\section{A. Approximately-finite memory}

Consider the model class of Definition 1 and the input class of Definition 2, Let $G$ denote the (convolution) operator defined by $G(s)$ and $H$ denote the operator defined by $(H v)(t)=h(v(t))$. It can be shown 2 , based on the stability of $G(s)$ and the Lipschitz property of $h$, that the map $(I+G H)^{-1}$ is well defined on $\mathcal{L}_{\infty}\left(\mathbb{R}_{+}\right)$. Thus,

$$
v=(I+G H)^{-1}\left(G i+g_{0}\right)
$$

where $g_{0} \in \mathcal{L}_{\infty}\left(\mathbb{R}_{+}\right)$is a term taking into account the exponentially decaying initial conditions of the linear system.

Let $G_{c \ell}$ denote the restriction of $(I+G H)^{-1} G$ to $\mathcal{U}$ (under zero initial conditions, this is the map from the input $i$ to the output $v$ ). We are interested in the following property.

Definition 3 ([9]). Let $F: \mathcal{U} \rightarrow \mathcal{L}_{\infty}\left(\mathbb{R}_{+}\right)$be a causal time-invariant operator. We say $F$ has approximately-finite memory with respect to $\mathcal{U}$, or $F \in \mathcal{A}(\mathcal{U})$, if for any given $\epsilon>0$, there is a $\Delta>0$ such that

$$
\left|(F u)(t)-\left(F W_{t, \eta} u\right)(t)\right|<\epsilon, \quad t \geq 0
$$

for all $\eta \geq \Delta$ and all $u \in \mathcal{U}$, where $W_{t, \eta}$ is the window operator

$$
\left(W_{t, \eta} u\right)(\tau)=\left\{\begin{array}{cc}
u(\tau), & t-\eta \leq \tau \leq t \\
0, & \text { otherwise }
\end{array}\right.
$$

${ }^{2}$ See e.g. [9, Section 2.3], where $(I+G H)^{-1}$ is denoted by $V$. 
The inequality (7) shows that the recent past of the input of a system in $\mathcal{A}(\mathcal{U})$ dominates the behavior of its output. An important result linking Definition 3 to the circle criterion is [9]. In our context, we have the following statement.

Proposition 1. Assume that one of the following two conditions are satisfied:

(i) $0 \leq \rho_{1}<\rho_{2}$, all poles of $G(s)$ are in $\operatorname{Re}[s]<0$, and $\operatorname{Re}[G(j \omega)] \geq 0$ for all $\omega \in \mathbb{R}$.

(ii) $\rho_{1}<0<\rho_{2}$, all poles of $G(s)$ are in $\operatorname{Re}[s]<0$, and the locus of $G(j \omega)$ for $-\infty<\omega<\infty$ is contained within the circle of radius $\left(\rho_{2}^{-1}-\rho_{1}^{-1}\right) / 2$ centered on the real axis of the complex plane at $-\left(\rho_{2}^{-1}+\rho_{1}^{-1}\right) / 2+j 0$.

Then $G_{c \ell}$ has approximately-finite memory on $\mathcal{U}$.

Proof. Let $\mathcal{U}^{\prime}$ be defined similarly to $\mathcal{U}$, but with $\xi^{\prime}=$ $\|G\|_{1} \xi$. Under our assumptions, [9, Theorem 1] ensures that the map $G H(I+G H)^{-1} \in \mathcal{A}\left(\mathcal{U}^{\prime}\right)$ (for simplicity, we denote operators and their restrictions by the same symbols). But since $G H(I+G H)^{-1}=I-(I+G H)^{-1}$, it follows from direct application of the inequality (7) that $(I+G H)^{-1}$ is also in $\mathcal{A}\left(\mathcal{U}^{\prime}\right)$. Thus, $G_{c \ell}$ is the cascade interconnection of $(I+G H)^{-1} \in \mathcal{A}\left(\mathcal{U}^{\prime}\right)$ with $G \in \mathcal{A}(\mathcal{U})$. Since $G u \in \mathcal{U}^{\prime}$ for all $u \in \mathcal{U}, G_{c \ell}$ can be shown to be in $\mathcal{A}(\mathcal{U})$ using the cascade interconnection resul 3 [8, Theorem 3].

\section{B. Linear output feedback}

If $h(v)$ possesses regions of negative conductance, i.e., $\rho_{1}<0$, and $G(s)$ fails to satisfy the circle condition (ii) of Proposition 11, the interconnection of Definition 11 might fail to belong to $\mathcal{A}(\mathcal{U})$ for any $\mathcal{U}$. In fact, we can argue that is the case for the two examples of Section $\amalg-A$. The Fitzhugh-Nagumo model, for instance, does not satisfy (7) for the input $i_{p}(t)=\left(\mu\left(t-t_{1}\right)-\mu\left(t-t_{2}\right)\right) \xi / 2$, with $\mu$ the Heaviside function and $t_{2}>t_{1}>0$. With zero initial conditions, this input can be used to drive the state of (2)-(3) away from an unstable equilibrium at the origin and towards a stable limit-cycle. As a consequence, for any constant $\eta$, (7) cannot hold for arbitrarily large $t>0$ and small $\epsilon$. A similar argument can be used for the Chua circuit, where the limit-cycle is replaced with a chaotic attractor.

The feedback law (6) can be used to endow the closed-loop operator with the approximately-finite memory property. To see this, note that the closed-loop feedback system with input $k v_{\mathrm{r}}$ and output $v$ can be described by the negative feedback interconnection of $G(s)$ with the static nonlinearity

$$
h_{k}(v)=h(v)+k v
$$

so that now we have

$$
\rho_{1}+k \leq \frac{h_{k}\left(v_{1}\right)-h_{k}\left(v_{2}\right)}{v_{1}-v_{2}} \leq \rho_{2}+k
$$

for all $v_{1} \neq v_{2}$.

Now, it is possible to make $\rho_{1}+k \geq 0$ by choosing $k>0$ large enough. Let $H_{k}$ denote the operator defined by $\left(H_{k} v\right)(t)=h_{k}(v(t))$, and consider the new closed-loop

\footnotetext{
${ }^{3}$ This result requires $(I+G H)^{-1}$ to be uniformly continuous on $\mathcal{L}_{\infty}\left(\mathbb{R}_{+}\right)$, which can be shown by means of [7, Corollary 3a].
}

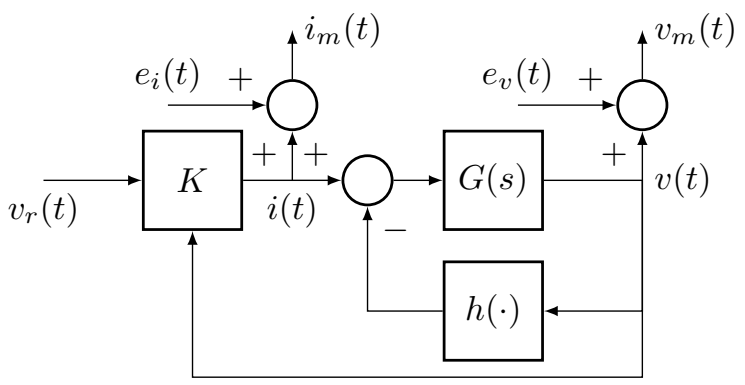

Fig. 2. Simplified output noise setup. $K=k\left(v_{r}-v\right)$ in the static identification stage, and $K=\kappa\left(v_{r}, v\right)$ from 18 the dynamic stage.

operator $G_{c \ell, k}=\left(I+G H_{k}\right)^{-1} G k$. Now (i) of Proposition 11 is satisfied, and we have $G_{c \ell, k} \in \mathcal{A}(\mathcal{U})$.

\section{A FEEDBACK IDENTIFICATION METHOD}

In this section, we show that it is possible to decouple the problem of identifying a nonlinear system belonging to the model class of Definition 1 into a nonlinear static identification stage and a dynamic mildly nonlinear identification stage. We work with the following simplifying assumption.

Assumption 1 (Simplified setup). The model class is described by Definition 1 In addition, $h$ is given by

$$
h(v)=a_{1} v+\sum_{j=2}^{J^{*}} a_{j} \phi_{j}(v)
$$

where $a_{j} \in \mathbb{R}$ and $J^{*} \in \mathbb{N} \cup\{\infty\}$. We assume the $\phi_{j}$ are known linearly independent functions which are Lipschitz continuous on every bounded subset of $\mathbb{R}$. The feedback law $i=k\left(v_{\mathrm{r}}-v\right)$, with $k+\rho_{1}>0$, is implemented with an ideal analog circuit. The signal $v_{r}$ is known, and the signals $i_{m}=i+e_{i}$ and $v_{m}=v+e_{v}$ are observed, where $e_{i}$ and $e_{v}$ are independent Gaussian coloured zero-mean noise terms with finite variances. Figure 2 with the block $K=k\left(v_{r}-v\right)$ gives a representation of this setup.

\section{A. Static identification stage}

We start by introducing the following concept.

Definition 4. We define the inverse static input-output characteristic by

$$
i_{\infty}(v)=\frac{1}{G(0)} v+h(v)
$$

where $G(0)>0$ by assumption.

The characteristic $i_{\infty}(v)$ gives the (unique) constant input required to establish an equilibrium at each constant $v$. Notice that under Assumption 11, estimating $i_{\infty}$ effectively amounts to estimating the nonlinear terms of $h$ in (10).

To estimate $i_{\infty}$, we need to stabilize the system at different steady-states $\bar{v}$. We ensure this by means of the output feedback (6). The equilibrium of the system must satisfy

$$
-\frac{1}{G(0)} v+k v_{\mathrm{r}}=h(v)+k v
$$


By assumption, the right-hand side of (12) is monotonically increasing. Since $h$ is continuous, it follows that (12) has a single solution $\bar{v}$ for every $\bar{v}_{\mathrm{r}}$. The fact that the system settles to the unique $\bar{v}$ when subject to a constant $\bar{v}_{\mathrm{r}}$ is guaranteed by the approximately-finite memory property [8, Theorem 2]. This can be alternatively be shown using the circle criterion [3, Theorem 7.2].

We can now discuss how to estimate $i_{\infty}$. A simple procedure begins by choosing a sufficiently large $k>0$ and a grid of $M$ constant values for $\bar{v}_{\mathrm{r}}$. Assume this grid is contained in the vector $\bar{V}_{\mathrm{r}}$. For each $m=1, \ldots, M$, we apply the input $\bar{V}_{\mathrm{r}}[\mathrm{m}]$ to the closed-loop system and wait for the system to settle to a corresponding output equilibrium $\bar{V}[m]$. This yields (as $t \rightarrow \infty$ ) an $M$-dimensional vector of true output steady-state values $\bar{V}$. In practice, the noise assumptions allow us to obtain consistent estimates $\hat{v}$ for $\bar{v}$ and $\hat{\imath}$ for $i_{\infty}(\bar{v})$ by averaging the measurements,

$$
\hat{v}_{N}=\frac{1}{N} \sum_{n=1}^{N} v_{m}\left(n T_{s}\right), \quad \hat{\imath}_{N}=\frac{1}{N} \sum_{n=1}^{N} i_{m}\left(n T_{s}\right)
$$

where $T_{s}$ is the measurement sampling period and $N$ is the number of samples. This yields estimate vectors $\hat{V}_{N}$ and $\hat{I}_{N}$.

Considering Assumption 1, a natural estimator for $i_{\infty}$ is

$$
\hat{\imath}_{\infty}(v)=w_{1} v+\sum_{j=2}^{J} w_{j} \phi_{j}(v)
$$

where $w_{j}$ are the estimator parameters, and $J \in \mathbb{N}$ is such that $J \leq M$. In order to estimate these parameters, we construct a matrix $\Phi_{N, J} \in \mathbb{R}^{M \times J}$ whose $m^{\text {th }}$ row is given by

$$
\left(\hat{V}_{N}[m], \phi_{2}\left(\hat{V}_{N}[m]\right), \ldots, \phi_{J}\left(\hat{V}_{N}[m]\right)\right)
$$

Assume that $\Phi_{N, J}$ has full rank. This can be accomplished by choosing a sufficiently wide and fine grid for the elements of $\bar{V}_{\mathrm{r}}$. Then, a parameter estimate $\hat{W}=\left(\hat{w}_{1}, \ldots, \hat{w}_{J}\right)^{T}$ is obtained by solving

$$
\min _{W} \sum_{m=1}^{M}\left(\hat{I}_{N}[m]-\hat{\imath}_{\infty}\left(\hat{V}_{N}[m]\right)\right)^{2}
$$

which yields

$$
\hat{W}_{N, J}=\left(\Phi_{N, J}^{T} \Phi_{N, J}\right)^{-1} \Phi_{N, J}^{T} \hat{I}_{N}
$$

We thus have that, as $N \rightarrow \infty$ and $J \rightarrow J^{*}$, as long as $\Phi_{N, J}$ has full column rank for all $J, \hat{\imath}_{\infty}(v)$ converges to $i_{\infty}(v)$, and each $\hat{w}_{j}$ converges to $a_{j}$ for $j=2,3, \ldots, J^{*}$ (we drop the subscripts $N$ and $J$ of $\hat{w}_{j}$ for clarity).

\section{B. Dynamic identification stage}

The main idea in the dynamic identification stage is to use the input

$$
i=\kappa\left(v, v_{\mathrm{r}}\right) \triangleq k\left(v_{\mathrm{r}}-v\right)+\sum_{j=2}^{J} \hat{w}_{j} \phi_{j}(v)
$$

so as to linearize the system by feedback.
Assumption 2. The feedback law (18) is implemented with an ideal analog circuit. The setup of the problem is represented by Figure 2, with $K=\kappa\left(v, v_{\mathrm{r}}\right)$ given by (18).

From the analysis in the previous section, as $N \rightarrow \infty$ and $J \rightarrow J^{*}$, the identification problem becomes one of identifying a linear system with input $v_{\mathrm{r}}$, output $v$, and an output error structure. The ground truth model, at those limits, is given by $G_{k}(s)=k G_{a}(s) /\left(1+k G_{a}(s)\right)$, with $G_{a}(s)=G(s) /\left(1+a_{1} G(s)\right)$. The system $G_{a}(s)$ lumps together the term $a_{1} v$ and the transfer function $G(s)$, which are indistinguishable from each other from the input-output perspective. The resulting linear identification problem is a well-known one for which consistency guarantees can be obtained with a variety of methods [4].

In practice, obviously, $N$ and $J$ will be finite, and the nonlinearity will not be perfectly canceled by feedback. In that case, identifying the closed-loop system from $v_{\mathrm{r}}$ to $v$ amounts to identifying a mildly nonlinear system that has an approximately-finite memory and is subject to output noise. The Best Linear Approximation (BLA) framework [10] ensures in this setting that, by using linear identification methods which are based on minimizing a squared sum of output residuals, we can obtain (asymptotically) an optimal approximation of the nonlinear system. Optimality, in this case, is defined with respect to the assumed input class [12]. Furthermore, due to the fact that operators with approximately-finite memory map periodic inputs to asymptotically periodic outputs [8, Theorem 9], by choosing periodic exciting signals, we can mitigate noise effects in the output by averaging the signal $v_{m}$ over different periods.

Given a best linear estimate $\hat{G}_{k}(s)$, to recover the estimate of the original nonlinear system with input $i$ and output $v$, we first compute

$$
\hat{G}_{a}(s)=\frac{1}{k} \frac{\hat{G}_{k}(s)}{1-\hat{G}_{k}(s)},
$$

which is necessary to account for the $k\left(v_{\mathrm{r}}-v\right)$ term in (18). The identified nonlinear system is then given by interconnecting, in negative feedback, the transfer function $\hat{G}_{a}(s)$ and the nonlinearity $\hat{h}(v)=\sum_{j=2}^{J} \hat{w}_{j} \phi_{j}(v)$.

\section{Simulations With a Realistic SETUP}

In a more realistic identification setting, the user-defined feedback loop around the physical system is implemented in discrete-time, and output measurement noise is fed back into the system dynamics.

In this section, using numerical simulations, we naively apply the procedure described in Section IV to identify the two systems from section II-A assuming the realistic setup of Figure 3 We assume that $e_{v}$ and $e_{i}$ are given by white Gaussian noise with the same variance, denoted by $\sigma$. With this, we aim to provide a proof of concept that the method still performs well in a realistic scenario.

We briefly describe the simulation procedure. Given a vector $\bar{V}_{\mathrm{r}}$, each of the $M$ experiments of the static identification stage was simulated by numerically integrating the dynamics 


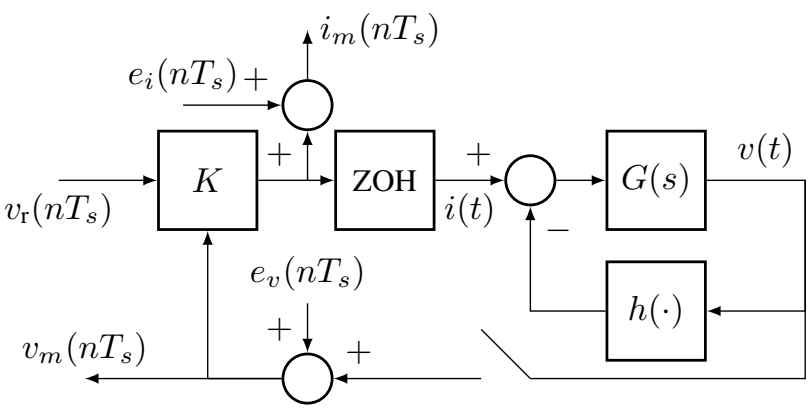

Fig. 3. Realistic identification setup. $K=k\left(v_{\mathrm{r}}-v\right)$ in the static static identification stage, and $K=\kappa\left(v_{\mathrm{r}}, v\right)$ from 18 in the dynamic stage. The block $\mathrm{ZOH}$ is a standard zero-order hold.

of the scheme shown in Figure 3, with $K$ given by $k\left(v_{\mathrm{r}}-v\right)$, with the input $v_{\mathrm{r}}(t)=\bar{V}_{\mathrm{r}}[m], t \geq 0$, and with zero initial conditions. Numerical integration was carried out for 100 seconds, which was sufficient to see (13) converge.

To generate data for the dynamic identification stage, we performed $R$ simulations ${ }^{4}$ corresponding to $R$ realizations of two periods the random-phase multisine inputs given by

$$
v_{\mathrm{r}}\left(n T_{s}\right)=\sum_{\ell=-N_{f}}^{N_{f}} u_{\ell} \sin \left(\frac{2 \pi}{N} \ell n+\theta_{\ell}\right), \quad n=0,1,2, \ldots
$$

where the $\theta_{\ell}$ are random variables uniformly distributed over $\left[0,2 \pi\left[, N=T / T_{s}\right.\right.$ is the number of samples per signal period $T$, and $N_{f}=f_{\max } T<N / 2$ is the harmonic number corresponding to the largest frequency in the signal, $f_{\max }$. The coefficients $u_{\ell}$ are chosen such that $u_{0}=0$ and $u_{\ell}=\bar{u}$, with $\bar{u}$ a constant used to set the input RMS level. Simulations were carried out by numerically integrating the dynamics of the scheme shown in Figure 3, with zero initial conditions, and with $K$ given by $\kappa\left(v_{\mathrm{r}}, v\right)$ in (18).

Using the generated data, a continuous-time transfer function $\hat{G}_{k}(s)$ was estimated using the off-the-shelf Matlab System Identification Toolbox 5 routine test 6 . The number of poles and zeros of the identified transfer were constrained to be the same as those of the ground truth ones. The identified linear model is recovered as in (19).

The results to be discussed next were obtained with data generated using the parameters in Table \ The signal-to-noise ratio (SNR) value refers to ratio of the average power of the output of the noiseless system in the dynamic identification stage, and the noise variance $\sigma^{2}$.

\section{A. Fitzhugh-Nagumo circuit}

Using the basis functions $\phi_{j}(v)=v^{j}, j=2,3$, Figure 4 shows that assuming a realistic setting results in a small error

\footnotetext{
${ }^{4}$ The simulations were performed in Matlab's Simulink ${ }^{\mathrm{TM}}$ using the numerical integration routine ode $15 \mathrm{~s}$ with a maximum step set to $10^{4}$ seconds and relative/absolute tolerances set to $10^{-6}$.

${ }^{5}$ Toolbox version 9.9, Matlab version R2018b.

${ }^{6}$ The function tfest was used with standard settings. The routine initializes parameters through the Instrument Variable (IV) method, and updates the parameters by minimizing a weighted prediction error norm using a nonlinear least-squares search method.
}

TABLE I

PARAMETERS USED IN THE GENERATION OF DATA.

\begin{tabular}{|c|c|c|c|c|c|c|c|}
\hline & $T_{s}$ & $\mathrm{k}$ & $f_{\max }$ & $R$ & $T$ & $\sigma$ & SNR \\
\hline FHN & $10^{-3} \mathrm{~s}$ & 1.5 & $100 \mathrm{~Hz}$ & 5 & $500 \mathrm{~s}$ & 0.01 & $40 \mathrm{~dB}$ \\
\hline Chua & $10^{-3} \mathrm{~s}$ & 5 & $100 \mathrm{~Hz}$ & 5 & $500 \mathrm{~s}$ & 0.01 & $40 \mathrm{~dB}$ \\
\hline
\end{tabular}
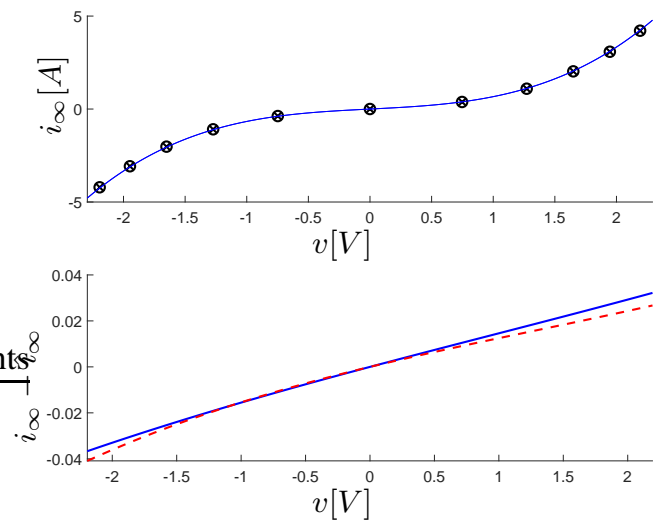

Fig. 4. Estimation of $i_{\infty}$ for the FHN circuit. Top: ground truth $i_{\infty}$ (line), estimates $(\hat{v}, \hat{\imath})$ with $\sigma=0.01$ (crosses) and $\sigma=0.1$ (circles). Bottom: error $i_{\infty}-\hat{\imath}_{\infty}$ with $\sigma=0.01$ (solid) and $\sigma=0.1$ (dashed).

$\left(i_{\infty}-\hat{\imath}_{\infty}\right)(v)$. The error remains roughly the same when the noise variance is increased by a factor of 10 .

Figure 5 shows validation of the identified model in closed-loop. For validation purposes, the mean of the input $i(t)$ was set to -1.5 , which puts the FHN system in the excitable regime, and results in a characteristic spiking behavior. It can be seen that the error is kept low for most of the time, except at moments when the model "misses" a spike. These misses occur due to the ultrasensitivity of excitable systems with respect to their inputs.

\section{B. Chua's circuit}

To capture the nonlinear components of a piecewiselinear nonlinearity such as (5), we use the basis functions $\phi_{2}(v)=\max \{0, v-1\}$ and $\phi_{3}(v)=\max \{0,-(v+1)\}$. Figure 6 shows the resulting nonlinearity estimation error.
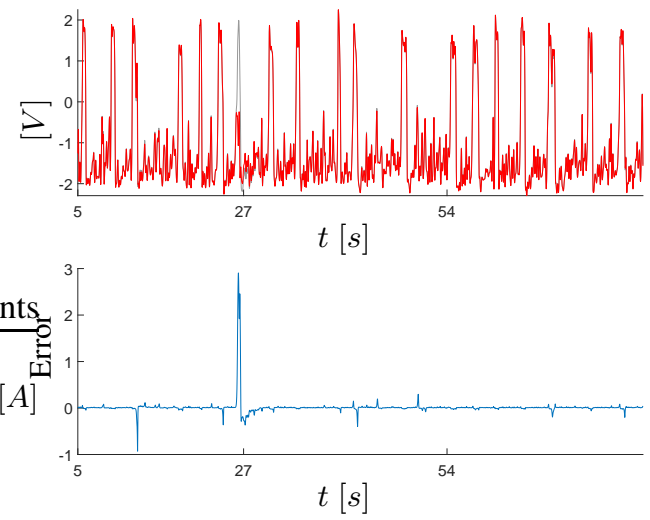

Fig. 5. Validation of the identified FHN circuit. Top: Ground truth model output $v(t)$ (gray) and identified model output $\hat{v}(t)$ (red). Middle: Output error $v(t)-\hat{v}(t)$. NRMSE $\approx 0.84$ for the interval shown. The NRMSE increases to about 0.97 when only data from $t \geq 30$ is taken into account: most of the error comes from the "missed spike" around $27 \mathrm{~s}$. 

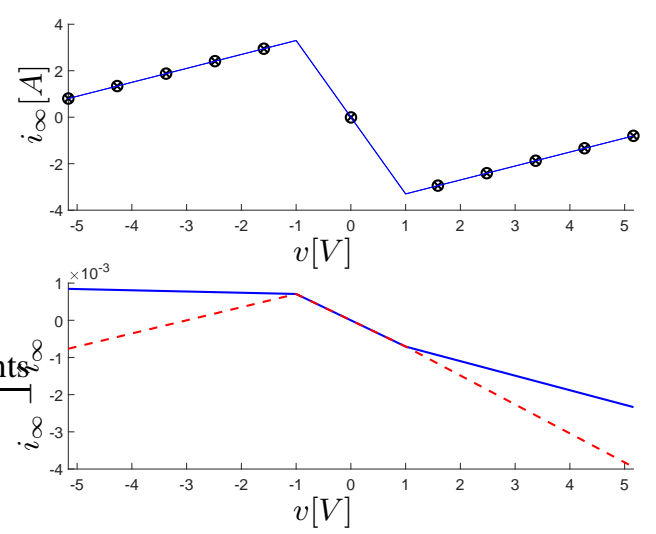

Fig. 6. Estimation of $i_{\infty}$ for the Chua circuit. Top: ground truth $i_{\infty}$ (line), estimates $(\hat{v}, \hat{\imath})$ with $\sigma=0.01$ (crosses) and $\sigma=0.1$ (circles). Bottom: error $i_{\infty}-\hat{\imath}_{\infty}$ with $\sigma=0.01$ (solid) and $\sigma=0.1$ (dashed).
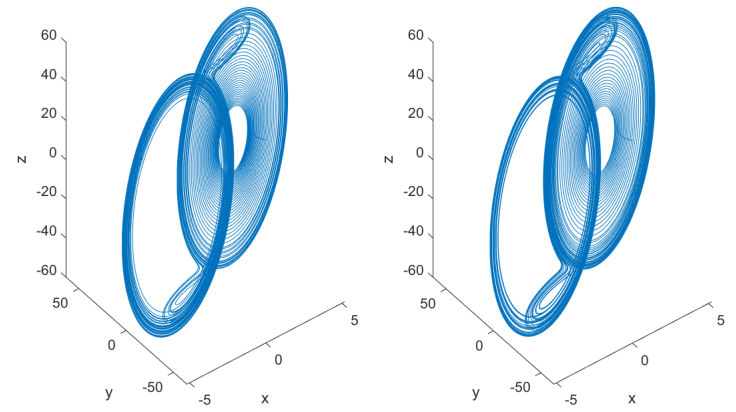

Fig. 7. Attractors of the ground truth Chua circuit (left) and of the identified Chua circuit (right). The trajectories in the states $(x, y, z)$ are obtained with a modal canonical state-space realization of the original $G_{a}(s)=$ $G(s) /(1-4 G(s))$ and of the estimated $\hat{G}_{a}(s)$.

Again, a tenfold increase in measurement noise does not severely affect the error.

To compare the complete identified model with the ground truth model, we first realize the linear dynamics of each system (lumped with the linear component of $h$ ) in the modal canonical state-space form. Starting from a nonzero initial condition, the resulting trajectories are shown in Figure 7. It can be seen that the "double-scroll" attractors are qualitatively very similar.

\section{Discussion}

It can be argued that the choice of the feedback gain $k$ is key to the success of the identification procedure developed in Section IV when it is applied to the more realistic case dealt with in this section. In principle, $k$ does not need to exceed $\left|\rho_{1}\right|$ by a very large margin, and indeed we chose it to be only slightly larger than $\left|\rho_{1}\right|$ in both simulations above. Choosing a suitable $k$ in this case can be viewed as part of experiment design. While our choices were good enough to avoid issues with the measurement noise that is fed back into the system, it is clear that difficulties might arise for systems with a large $\left|\rho_{1}\right|$. If that is the case, and if it is possible, analog feedback should be used.

\section{CONCLUSION AND FUture WORK}

In this paper, we have observed that feedback can simplify the identification of a nonlinear system. We have illustrated this idea with the elementary situation where the original system is the feedback interconnection of a passive LTI system and a static nonlinearity. In this case, the use of output feedback as part of experiment design provides a straighforward solution to the problem. This procedure is sufficient to identify nonlinear behaviors such as excitability (Fitzugh-Nagumo) or chaos (Chua).

It is important to mention that this method can be used as a means to obtain initial estimates for a final identification stage [12], where we perform nonlinear optimization of the simulation error of the nonlinear feedback system. In this stage, consistency guarantees can be obtained depending on the noise setting.

In future research, we aim to generalize the method to neuronal conductance-based models [2], in which case the fading memory element is dynamic rather than static.

\section{REFERENCES}

[1] S. Boyd and L. Chua, "Fading memory and the problem of approximating nonlinear operators with Volterra series," IEEE Transactions on Circuits and Systems, vol. 32, no. 11, pp. 1150-1161, Nov. 1985.

[2] A. L. Hodgkin and A. F. Huxley, "A quantitative description of membrane current and its application to conduction and excitation in nerve," The Journal of Physiology, vol. 117, no. 4, pp. 500-544, Aug. 1952.

[3] H. K. Khalil, Nonlinear Systems, 3rd ed. Upper Saddle River, NJ: Prentice Hall, 2002.

[4] L. Ljung, System Identification: Theory for the User. Upper Saddle River, NJ: Prentice Hall PTR, 1999.

[5] T. Matsumoto, "A chaotic attractor from Chua's circuit," IEEE Transactions on Circuits and Systems, vol. 31, no. 12, pp. 1055-1058, Dec. 1984.

[6] J. Nagumo, S. Arimoto, and S. Yoshizawa, "An Active Pulse Transmission Line Simulating Nerve Axon," Proceedings of the IRE, vol. 50, no. 10, pp. 2061-2070, Oct. 1962.

[7] I. W. Sandberg, "Some results on the theory of physical systems governed by nonlinear functional equations," The Bell System Technical Journal, vol. 44, no. 5, pp. 871-898, May 1965.

[8] — - "Approximately-finite memory and input-output maps," IEEE Transactions on Circuits and Systems I: Fundamental Theory and Applications, vol. 39, no. 7, pp. 549-556, Jul. 1992.

[9] - "Approximately finite memory and the circle criterion," IEEE Transactions on Circuits and Systems I: Fundamental Theory and Applications, vol. 41, no. 7, pp. 473-476, Jul. 1994.

[10] J. Schoukens, R. Pintelon, T. Dobrowiecki, and Y. Rolain, "Identification of linear systems with nonlinear distortions," Automatica, vol. 41, no. 3, pp. 491-504, Mar. 2005.

[11] M. Schoukens and Y. Rolain, "Parametric Identification of Parallel Wiener Systems," IEEE Transactions on Instrumentation and Measurement, vol. 61, no. 10, pp. 2825-2832, Oct. 2012.

[12] M. Schoukens and K. Tiels, "Identification of block-oriented nonlinear systems starting from linear approximations: A survey," Automatica, vol. 85, pp. 272-292, Nov. 2017.

[13] R. Sepulchre, G. Drion, and A. Franci, "Excitable Behaviors," in Emerging Applications of Control and Systems Theory. Springer International Publishing, Jan. 2018, pp. 269-280. 